\title{
Orthodontic debonding: five-minute fallow times a possibility?
}

\section{Quantitative analysis of particulate matter release during orthodontic procedures: A pilot study. Br Dent J 2020; 229: http://dx.doi.org/10.1038/s41415-020-2280-5.}

Transmission of SARS-CoV-2 through aerosol is currently a major concern in the provision of dental care. It is widely accepted that aerosols are produced during a range of dental procedures which pose a potential threat to dental professionals and patients. This has led to enhanced

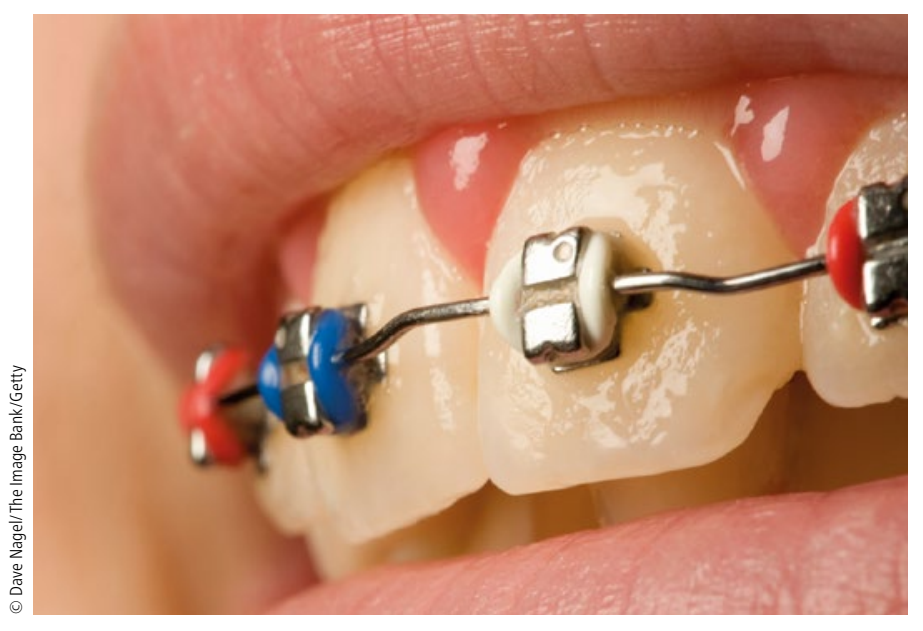

infection prevention and control procedures, PPE requirements and implementation of fallow times in dental practices. However, there has been little consideration on aerosol generation during orthodontic procedures and their associated mitigations. In order to investigate this, a pilot study was conducted to determine the potential risk of SARS-CoV-2 transmission from aerosol produced during orthodontic procedures including handpiece-based debonding and the use of 3-in-1 air-water syringes.

Orthodontic debonding procedures involving composite-based adhesive removal were simulated using a dental manikin with acrylic teeth, in a closed side-surgery with an air exchange system. A 1:1 contraangle handpiece and fast hand-piece with variation in air and water flow were used. Removal of acid-etch was also simulated with combined 3-in-1 air-water syringe. All experiments were carried out by the same operator and with high volume suction. An optical particle sizer and a scanning mobility particle scanner were used to assess particulate matter with measurements starting ten minutes before the procedure to assess baseline concentrations of particulate matter. A single SARSCoV-2 viron ranges from $0.08-0.1 \mu \mathrm{m}$ in diameter and so information on particles less than $0.08 \mu \mathrm{m}$ was discarded. Particle counts were combined into four different categories: 'very small' $(0.08-0.26 \mu \mathrm{m})$, 'small' $(0.26-0.9 \mu \mathrm{m})$, 'medium' $(0.902-2.7 \mu \mathrm{m})$ and 'large' $(2.7-10 \mu \mathrm{m})$.

Most procedures led to a statistically significant increase in aerosol concentration in most size ranges. The results showed that:

- Standard debonding procedures involving air but no water were associated with increase in the 'very small' and 'small' particles and reduced to baseline levels over approximately five minutes

- Debonding without air produced similar levels of aerosols as standard debonding procedures

- Debonding with water produced large increases in aerosol levels with particles of all sizes produced throughout the experiment

- Debonding with water and a fast handpiece led to the most significant increase in particles

- Combined use of the 3-in-1 air-water syringe to remove acid-etch did not result in any detectable increase in aerosol levels.

The authors concluded that particulate matter was consistently released during orthodontic debonding, although the concentration and volume of matter produced using the slow handpiece was markedly less than associated with use of a fast handpiece. Therefore, the authors recommend in current SARS-CoV-2 risk environments, orthodontic debonding be carried out with a slow handpiece and without the use of water as an irrigant to mitigate the risk of infection to staff and patients. In this setting, fallow times can be reduced to approximately five minutes and the use of the 3-in-1 air-water syringe be considered as a low risk dental procedure. However, further research is required as this fallow period may not be true for open environments and where air filtration facilities are not available.

By Iqra Chawdhary, Forest Hill Dental Surgery, UK. 